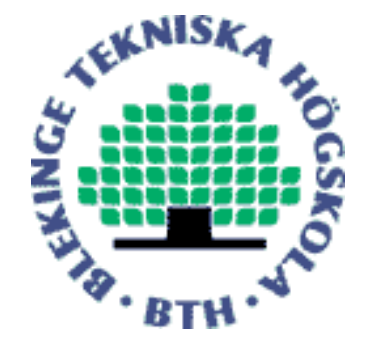

Copyright (C) 2014 IEEE.

Citation for the published paper:

A method to implement SAR slow-time stap in beamforming stage of fast backprojection algorithm

Viet Thuy Vu, Mats Pettersson, Svante Björklund

\title{
IGARSS
}

2014 Quebec City

This material is posted here with permission of the IEEE. Such permission of the IEEE does not in any way imply IEEE endorsement of any of BTH's products or services Internal or personal use of this material is permitted. However, permission to reprint/republish this material for advertising or promotional purposes or for creating new collective works for resale or redistribution must be obtained from the IEEE by sending a blank email message to pubs-permissions@ieee.org.

By choosing to view this document, you agree to all provisions of the copyright laws protecting it. 


\section{A METHOD TO IMPLEMENT SAR SLOW-TIME STAP IN BEAMFORMING STAGE OF FAST BACKPROJECTION ALGORITHM}

\author{
Viet T. Vu, Mats I. Pettersson* \\ Blekinge Institute of Technology \\ Campus Gräsvik, 37179 Karlskrona, Sweden
}

\author{
Svante Björklund \\ Swedish Defence Research Agency \\ P.O. Box 1165, 58111 Linköping, Sweden
}

\begin{abstract}
This paper presents a method to implement SAR slow-time space time adaptive processing (STAP) in the beamforming stage of the fast backprojection algorithm. This method is different from the recently published method where the SAR fast-time STAP is implemented after the beamforming stage for detection and then imaging. As a common SAR STAP method, the method proposed in this paper can be used for ground moving target indication (GMTI) and reconstruction of the image of the detected moving targets. The paper also presents some simulation results in order to illustrate the proposed method.
\end{abstract}

Index Terms - space-time adaptive processing, backprojection, detection, moving target

\section{INTRODUCTION}

Ground moving target indication (GMTI) is possible for radars based on antenna array solutions, however, without focus on imaging capability. Synthetic aperture radar (SAR) shows not only the capability in GMTI but also reconstruction of target's image after the detection and the estimation stages. This makes SAR more and more important for GMTI.

Implementation of SAR space time adaptive processing (STAP) for GMTI has been investigated recently. A method which combines fast backprojection SAR processing methods with moving target detection using space time processing has been developed in [1]. This method has also been experimented with LORA GMTI data in [2] giving good results in terms of GMTI. Due to the sampling requirements of the SAR algorithm considered in the method, the computational load of the algorithm without STAP is already high for even UHF-band. An integration of the displaced phase center antenna (DPCA) [3] and the fast-time STAP techniques into another fast backprojection algorithm (local processing which is presented in section 2) has been proposed in [4]. The proposal is declared to lighten the strict requirements of DPCA

\footnotetext{
${ }^{*}$ The authors would like to thank the KK-Foundation for the financial support in this research project, Swedish Defence Research Agency, Saab Bofors Dynamics, Saab Electronic Defence Systems and RUAG Space for their cooperation.
}

and improve the practicality of STAP. However, the strict requirements of DPCA such as the straight flight track, constant platform speed and identical channels are still the obstacles to apply this technique universally. In addition, DPCA is quite sensitive to the speed of the moving targets. The subtraction in DPCA can suppress the ground clutter but can also raise the noise level significantly. The method introduced in [4] allows a significant computational reduction since the fasttime STAP is implemented after the beamforming stage of the fast backprojection algorithm [5]. Hence, the dimension of the covariance matrix in STAP is reduced from the square of number of SAR data samples to the square of number of beam samples. If we select small subimages, this reduction is significant. The number of operations required by STAP is also reduced by a factor of subaperture and this reduction is also significant if we select long subapertures. However, if we perform the SAR slow-time STAP in the beamforming stage of the fast backprojection algorithm, the dimension of the covariance matrix can further be reduced as it is proportional to the subaperture length. In this case, a selection of short subaperture is recommended. GMTI can be handled similar to a traditional radar STAP.

The goal of this paper is to present a method to implement SAR slow-time STAP on the beam forming stage of the fast backprojection algorithm. With the method, we try to minimize the processing time as the dimension of the covariance matrix can be selected. As shown latter, the dimension of the covariance matrix in this case is proportional to the selected subaperture and the number of SAR channels which is taken into account.

\section{BEAMFORMING STAGE IN FAST BACKPROJECTION ALGORITHM}

The fast backprojection algorithm considered in this paper refers to the algorithm with local processing, i.e. the reconstruction of a SAR scene from a SAR data set is processed on a subaperture and subimage basis [5]. According to this principle, the full aperture associated with the SAR data set is split into a number of shorter subapertures while the whole SAR image area is segmented into smaller subimages. Please 
note that the selections of subapertures and subimages depend strongly on SAR system parameters, required image quality and processing time. However, any selection must ensure the phase error to be smaller than $\pi / 8$ (far field condition). The processing is carried out in two stages, beamforming and backprojection with certain approximations. Please note that the concept of beamforming in the fast backprojection algorithm is different from the common concept of beamforming which is widely used in the antenna technique.

In the beamforming stage, a beam corresponding to a subimage is formed by a superposition of SAR data belonging to that subaperture which has been shifted in time with respected to the centers of the subaperture and the subimage. If we call $g(t, \tau)$ the range-compressed SAR data where $t$ and $\tau$ are slow- and fast time samples, respectively, and $\Theta(\tau)$ is the beam formed by the superposition of SAR data, the mathematical expression for the beam forming stage can be given by

$$
\Theta_{m, k}(\tau)=\int_{t_{m}-\frac{t_{M}}{2}}^{t_{m}+\frac{t_{M}}{2}} g\left(t, \tau+\tau_{m, k}\right) d t
$$

where $m$ and $k$ indicates the orders of subaperture and subimage, respectively, $t_{M}$ is the integration time and corresponds to the number of aperture positions in one subaperture, and $\tau_{m, k}$ is the shift in time with respected to the centers of the $m$-th subaperture and the $k$-th subimage.

In the next stage, the beam $\Theta_{m, k}(\tau)$ formed in the previous stage is approximately backprojected to the $k$-th subimage. This procedure will be repeated for all defined subapertures and subimages. The image of the SAR scene is retrieved by arranging the subimages in a correct order.

\section{SPACE TIME ADAPTIVE PROCESSING}

The suppression of ground clutter and noise determines the possibility to detect and to image moving targets. The ground clutter is interpreted as the radar backscattering in the SAR scene while the noise is thermal noise originated from the environment and the radar system. Let's have a look again at the theory of adaptive radar developed in [6]. According to Theorem 1 , to detect signal $\mathbf{s}$ in noise $\mathbf{n}$, the signal plus noise $\mathbf{g}=(\mathbf{s}+\mathbf{n})$ is put thought a filter $\mathbf{w}$ as

$$
\Theta=\mathbf{w}^{\mathrm{T}} \mathbf{g}
$$

where $\mathrm{T}$ denotes matrix transpose. The filter $\mathbf{w}$, which gives the maximum probability of detection for a fixed probability of false alarm, is shown to be

$$
\mathbf{w}=\kappa \mathbf{R}^{-1} \mathbf{s}^{*}
$$

where $\kappa$ is a nonzero complex number, $\mathbf{R}$ the covariance matrix of noise and the asterisk complex conjugation operation.
If $\mathbf{s}$ in (2) is a steering vector, main-beam clutter will be minimized or cancelled [2]. The covariance matrix is defined by

$$
\mathbf{R}=\mathfrak{E}\left\langle\mathbf{n}^{*} \mathbf{n}^{\mathrm{T}}\right\rangle
$$

where $\mathfrak{E}\langle\rangle$ denotes the expectation operation. In this paper, we assume that $I$ training data $\mathbf{z}_{i}$ (containing only $\mathbf{n}$ ) is available for the covariance matrix estimation. An estimated version of (4) can be expressed in the foloiwng form

$$
\tilde{\mathbf{R}}=\frac{1}{I} \sum_{i=1}^{I} \mathbf{z}_{i} \mathbf{z}_{i}^{\mathrm{H}}
$$

where $\mathrm{H}$ denotes Hermitian operator.

\section{STAP IMPLEMNETATION IN BEAMFORMING}

Let's consider a multichannel SAR system with an $N$ elements receiving uniformly spaced linear array (ULA). In processing of each channel, $M$ apeture positions correspoding to $M$ pulses are shifted in time and form a subaperture in the beamforming stage of the fast backprojection algorithm. The considered number of range samples is $L$. The dimension of the space-time data cube is therefore $N \times M \times L$. Since a slow time STAP is here considered, a complex snapshort of the space-time data is taken at the $l$-th range sample. The snapshort results in the vector $\mathrm{g}$ in (2) as follows

$$
\mathbf{g}=\left(\begin{array}{c}
g_{1}(m+1, l) \\
\vdots \\
g_{1}(m+M, l) \\
\vdots \\
g_{N}(m+1, l) \\
\vdots \\
g_{N}(m+M, l)
\end{array}\right)
$$

where $m$ and $l$ are slow- and fast time samples instead of slow time $t$ and fast time $\tau$. The vector $\mathbf{s}$ in (3) is here considered as a steering vector which allows minimizing main-beam clutter. It is the Kronecker product of a $N \times 1$ spatial steering vector $\mathbf{s}_{\mathbf{s}}$ and a $M \times 1$ temporal seering vector $\mathbf{s}_{\mathbf{t}}$

$$
\mathbf{s}=\mathbf{s}_{\mathbf{s}} \otimes \mathbf{s}_{\mathbf{t}}
$$

where the spatial steering vector is a function of angle of arrival (AOA) $\varphi$,

$\mathbf{s}_{\mathbf{s}}=\left(1, \exp \left[\mathrm{i} \frac{2 \pi d}{\lambda} \cos \varphi\right], \cdots, \exp \left[\mathrm{i} \frac{2 \pi(N-1) d}{\lambda} \cos \varphi\right]\right)^{\mathrm{T}}$

while temporal seering vector is a function of Doppler shift

$$
\mathbf{s}_{\mathbf{t}}=\left(1, \exp \left[\mathrm{i} \frac{4 \pi T \nu_{s}}{\lambda}\right], \cdots, \exp \left[\mathrm{i} \frac{4 \pi(M-1) T \nu_{s}}{\lambda}\right]\right)^{\mathrm{T}}
$$




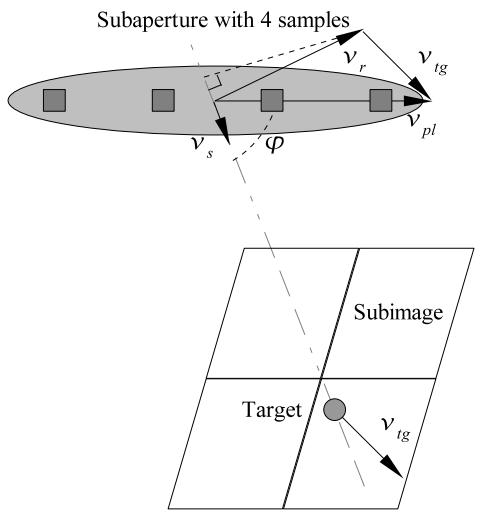

Fig. 1. An illustration for estimating steering vector.

In (8) and (9), $\lambda$ denotes the wavelength, $d$ the element space of ULA, $T$ pulse repetition interval which is the inverse of pulse repetition frequency (PRF), and $\vec{\nu}_{s}$ the radial velocity or line of sight velocity. The AOA $\varphi$ can be approximated by the angle formed by the platform flight track and the line between center of the subaperture and the center of the subimage and this angle is avalable.

One way to estimate the radial velocity is to use the concept of realtive velocity, i.e. the velocity of an object in the rest frame of another object. If a SAR platform move with a velocity $\vec{\nu}_{p l}$ while a target in a ground scene with another velocity $\vec{\nu}_{t g}$, then the relative velocity of the platform's velocity in the rest frame of the target's velocity is $\vec{\nu}_{r}=\vec{\nu}_{p l}-\vec{\nu}_{t g}$ as illustrated in Fig. 1. The relative speed and the direction of the relative velocity are defined by

$$
\nu_{r}=\sqrt{\nu_{p l}^{2}+\nu_{t g}^{2}-2 \nu_{p l} \nu_{t g} \cos \alpha}
$$

and

$$
\theta=\operatorname{acos}\left(\frac{\nu_{r}^{2}+\nu_{p l}^{2}-\nu_{t g}^{2}}{2 \nu_{r} \nu_{p l}}\right)
$$

respectively, where $\alpha$ is angle formed by the platform's velocity and the target's velocity. The radial speed can be shown to be

$$
\nu_{s}=\nu_{r} \cos (\varphi+\theta)
$$

We can perform the detection on the beam samples formed after STAP processing based on a defined detection threshold. Hence, instead of summing each $M$ rows of $\mathbf{g}$ in (6) to form the $l$-th samples of the beams of each channel as the common beamforming step, $g$ is put through the filter $\mathbf{w}$ where $\mathbf{s}$ is steering vector defined by (7) and $\mathbf{R}$ is estimated by (5) with the available training data. The dimension of $\mathbf{R}$ is $M \times N$ and we can select small subaperture, i.e. $M$, to optimize the processing time. The output of the filter is the single $l$-th sample of the beam.

The samples of the beam are then used for the second stage of the fast backprojection algorithm, which is presented in section 2, to form image of the detected moving target.
Table 1. LORA parameters.

\begin{tabular}{|c|c|}
\hline Parameter & Value \\
\hline Operating frequency range & $307 \mathrm{MHz}-333 \mathrm{MHz}$ \\
\hline Number of Tx/Rx antennas & $1 / 2$ \\
\hline Platform speed & $130 \mathrm{~m} / \mathrm{s}$ \\
\hline Flight altitude & $3000 \mathrm{~m}$ \\
\hline PRF & $1300 \mathrm{~Hz}$ \\
\hline Aperture step & $0.1 \mathrm{~m}$ \\
\hline Number of aperture positions & 6001 \\
\hline Minimum range & $\approx 12000 \mathrm{~m}$ \\
\hline
\end{tabular}

\section{SIMULATION RESULTS AND EVALUATION}

In this section, we present some results to illustrate the proposed method. The reference SAR system for the following simulations is LORA which is developed by Swedish Defense Research Agency. For GMTI purposes, this system is configured as a bistatic SAR system with three antennas (one transmits and two receive simultaneously) and uses the frequency range of $307.2-332.8 \mathrm{MHz}$ as given in Table 1. With the given element spacing, the displacement of the phase center of the forward and backward antenna is approximately $\lambda / 2$.

In the simulations, the ground SAR scene is simulated by a target which is point-like scatterer moving with the speed of $5 \mathrm{~m} / \mathrm{s}$ and the direction of $90^{\circ}$. The relative speed calculated with (10) and then normalized with respect to the speed of the platform is approximated to one. This means that the focusing is not significant and can be neglect. The radar cross section (RCS) of the target is normalized to $\sigma=1$. The ground clutter is simulated by random stationary point targets spread over all the ground scene. Their RCSs are given by the pseudorandom values drawn from the standard uniform distribution on the open interval $(0,4)$. The thermal noise is assumed to be AWGN with the noise level is $0 \mathrm{~dB}$. The SAR scene reconstructed with a single channel data is given in Fig. 2 where the clutter speads everywhere in the SAR image and hides the moving target which is the subject to detect. The SAR image is processed with the fast backprojection presented in section 2 and the beams is formed with the normal beamforming procedure as expressed by (1), i.e. only shifting in time and superposition. A beam formed with this procedure is ploted in Fig. 3. a. This beam coresponds to the data of the first apertures and the third subimage.

Let's continue to consider the SAR data coresponding to the first apertures and the third subimage. The dual channel SAR data is shifted in time with respect to the center of the first subapertures ( 2 subapertures of the dual channel in this case) and the third subimage. The shifted SAR data is then stacked in the form of the vector $\mathrm{g}$ given by (6). 


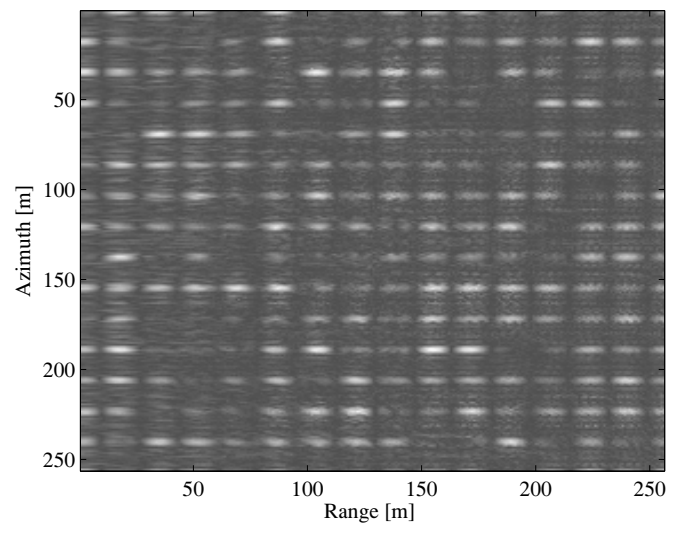

Fig. 2. SAR image of the simulated ground scene.
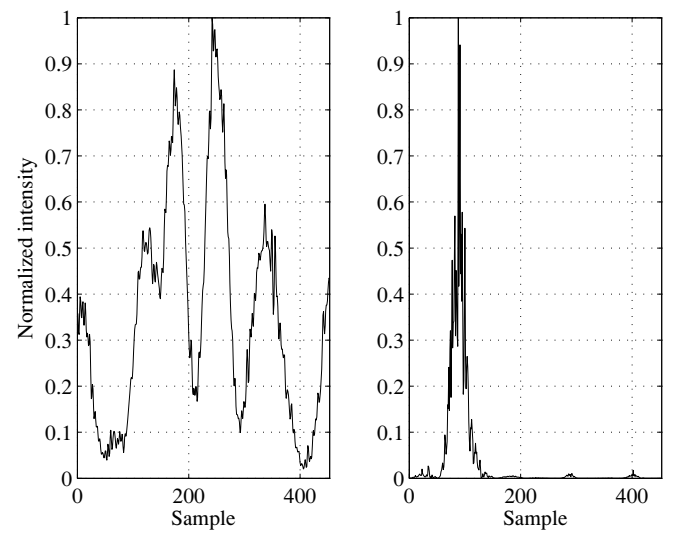

Fig. 3. Beam formed with (a) normal procedure, (b) STAP.

STAP beams are formed with the dual channel data by (2) where the filter is designed by (3). Please note that we use the steering vector (7) in the filter design. Fig. 3. b shows the STAP beam formed with the data of the first apertures and the third subimage.. In comparison to the beam given in Fig. 3. a, the clutter in the beam formed with STAP is suppressed significantly. Only the radar signal reflected from the moving target and the thermal noise remains in the beam. This implementation is carried out for all other subapertures and subimages.

The beams formed with STAP are used in the second stage of the fast backprojection algorithm in order to form the SAR image of the moving target. This image is given in Fig. 4. As observed, the clutter almost disappears in the new SAR image.

\section{CONCLUSION}

In this paper, we present a method to implement SAR slowtime STAP on the beam forming stage of the fast backpro-

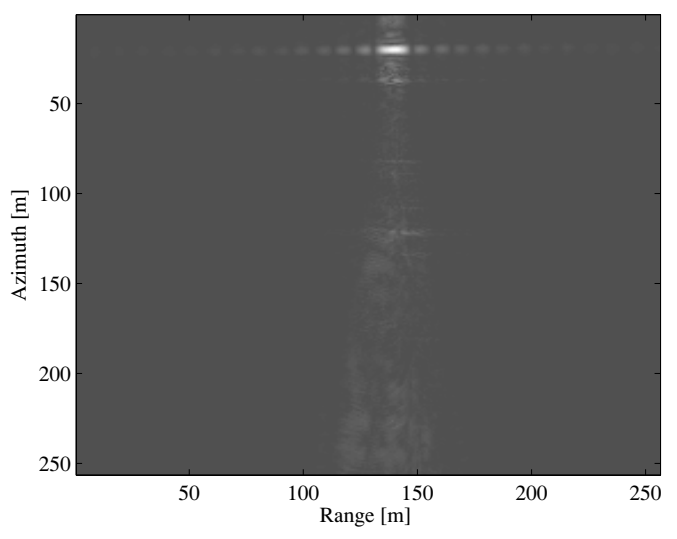

Fig. 4. SAR image of the moving target.

jection algorithm. As a common SAR STAP method, the method can be used for GMTI and reconstruction of the image of the detected moving targets. One of the good points of the method is that the method allows us to minimize the processing time as the dimension of the covariance matrix can be selected. The simulation results presented in this paper illustrate the method.

\section{REFERENCES}

[1] M.I. Pettersson, "Detection of moving targets in wideband SAR", IEEE Trans. Aerosp. Electron. Syst., vol. 40, no. 3, pp. 780-796, 2004.

[2] T.K. Sjögren, V.T. Vu, M.I. Pettersson, F. Wang, D.J.G Murdin, A. Gustavsson and L.M.H. Ulander, "Suppression of clutter in multichannel SAR GMTI", IEEE Trans. Trans. Geosci. Remote Sensing, accepted for publication 2013.

[3] M.I. Skolnik, Radar Handbook, 2nd ed. New York, NY: McGraw-Hill, 1990, ch. 16, pp. 8-17.

[4] V.T. Vu, T.K. Sjögren and M.I. Pettersson, "Integrating space-time processing into time-domain backprojection process for detection and imaging moving objects", in IEEE IGARSS 2010, Honolulu, HI, USA, July 2010, pp. 4106-4109.

[5] V.T. Vu, T.K. Sjögren and M.I. Pettersson, "Fast backprojection algorithm for UWB bistatic SAR", in in Proc. RadarCon 2011, Kansas City, MO, USA, May 2011, pp. 431-434.

[6] L.E. Brennan, L.S. Reed, "Theory of adaptive radar" IEEE Trans. Aerosp. Electron. Syst., vol. AES-9, no. 2, pp. 237-252, 1973. 